\title{
A review of mental health literacy strategy for adolescence
}

\author{
Padmi Dhyah Yulianti ${ }^{1}$, Endang R. Surjaningrum ${ }^{2}$ \\ ${ }^{1}$ Doctoral Psychology Program, Faculty of Psychology, Airlangga University, Surabaya, Indonesia \\ ${ }^{1}$ Guidance and Counseling, Faculty of Education, PGRI Semarang University, Semarang, Indonesia \\ ${ }^{2}$ Faculty of Psychology, Airlangga University, Surabaya, Indonesia
}

\section{Article Info}

Article history:

Received Feb 3, 2021

Revised Aug 12, 2021

Accepted Aug 24, 2021

\section{Keywords:}

Adolescence

Literacy

Mental health

Strategy

\begin{abstract}
Adolescents' problems concerning mental health could a burden for individual, families, and environments. To prevent further worsening mental problems, mental health literacy is needed. Mental health literacy has been promoted in advanced countries. However, it is implemented optimally in developing countries. This research aimed to find out the mental health literacy strategies done by adolescents. This article is a systematic literature review study. To obtain the articles for analysis purposes, search engine assistance was used such as Cochrane Library, Medpub, and ERIC. From 82 collected articles, and inclusion and exclusion stages were carried out to select the articles for the literature study purpose. They were then extracted from individually to get the significant findings. From the results of 11 reviewed articles, a conclusion was found. There were different mental health literacy implementation strategies in each country. Various mental health strategy types for adolescents covered national, community, schoolbased, and self-empowerment scope strategies.
\end{abstract}

This is an open access article under the CC BY-SA license.

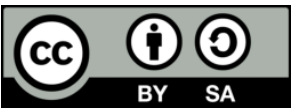

\section{Corresponding Author:}

Padmi Dhyah Yulianti

Faculty of Psychology

Airlangga University

4-6 Airlangga Road, Airlangga University, East Java, Indonesia

Email: yuli.dhy@gmail.com

\section{INTRODUCTION}

The adolescent is an sensitive periods [1] It was since on one side, adolescents are required to learn new behaviors and attitudes for the sake of their future life. Meanwhile, on another hand, adolescents do not voluntarily leave their joyful behaviors in their childhood yet. This seeking personality condition of adolescents would influence their psychological conditions. If adolescents could overcome various challenges and demands in an integrated manner in their lives, then they would be able to find out their personalities. Those personalities would be brought by them until their adulthood. However, if adolescents fail, then they would be in a long critical condition.

Using NCS-A data, Kessler et al. estimated the 12-month prevalence of mental illness to be $40.3 \%$ among adolescent [2]. Mental health disorder sufferers for adolescents, many problems concerning depression and anxiety occur [3], [4]. Adolescents' problems are such as anxiety and depression. They indirectly make adolescents passed away but those problems are troublesome. They could lead the sufferers to commit suicide due to the stress. For both children and adolescents, problems concerning mental health could be a burden for either individuals, families, and environments [5]. An adolescent that suffers mental health problems would have his academic interrupted such as depression, anxiety, and eating disorder [6]. Thus, paying attention to adolescents' mental health is the most important thing since they would influence significantly the adolescents' lives in the future, people aged 15-24 suffer mental health disorders [7]. 
To prevent a further worsening condition of mental health problems, an effort is required to reduce mental health disorders prevalences. One of the attempts to do is by promoting mental health literacy. The concept of mental health literacy was firstly proposed by in 1997. Mental health literacy in science and belief in recognizing problems, management, or prevention [8]. There were several components in mental health literacy such as specific problem recognition or difference of a psychological distress type, having proper knowledge and belief about certain risk factors and causes, having proper understanding and knowledge about self-help intervention, having knowledge and belief about professional assistance availability, having attitudes to seek accurate assistance, having the knowledge to seek information about mental health [9].

Adolescents need to have mental health literacy [10], [11]. Enhancing mental health literacy level should be considerd as an important preventive measures of mental health problem for young people [10]. Mental health literacy in adolescents has major implication s for early identification and intervention of mental health issues [12]. Since adolescents who have poor mental health literacy would also have lower skills in using the service [13].

Mental health literacy has been promoted in advanced countries [14], [15]. However, it is implemented optimally in developing countries. A bad literacy is concerned with low seeking assistance skill [16] and low skills to use service as social stigma and discriminative behavior [17]. Thus, this research aimed to find out the mental health literacy strategies done by adolescents.

\section{RESEARCH METHOD}

This article is a literature review study. This literature review aims to answer the proposed research questions to be more specific, structured, or systematic, planned and focused. High quality of Systematic Literature Review support better decisions for policy makers, entrepreneurs and researchers to synthesize the literature under review [18]. This literature review was conducted through identification, synthesis, and assessment of all provided evidence. It was done qualitatively and quantitatively so it could have strong and empirical answers related to the focused research questions [19]. A literature study helps the researcher; i) articulate cluar goals; ii) show the evidence of adeqquate preparation; iii) Select appropriate methods; iv) communicate relevant results; v) engage in reflective critique [20].

To obtain the articles for analysis purposes, search engine assistance was used such as the Cochrane library, Medpub, and ERIC. The search for the articles was done in August 2019. The search used keywords such as "mental health literacy", "adolescents", and "mental health literacy program". The use of these keywords was to keep the focus of the analyzed research objects. The researchers only used relevant articles with the research questions. In the next step, the researchers downloaded the articles completely to make the data comprehensive. Based on the search, the researchers successfully obtained the relevant findings with the research questions. Therefore, 82 articles were found in total. Inclusion and exclusion stages were carried out to select the articles for the literature study purpose. The researchers selected qualified articles. The articles should be complete (full paper). They should be inline between the abstracts and the contents. The articles also should provide a scope of the research. This limitation was done to get the novelty of the research. They were then extracted from individually to get the significant findings. The data extraction consisted of authors, country, sample, and the applied strategy.

\section{RESULTS AND DISCUSSION}

There were eleven relevant articles with the research questions. On the researcher's side, each article involved members of about two to twelve persons. The respondents' numbers consisted of students or adolescents with the lowest number of 271 and the highest number of 5,399. The countries consisted of : Indonesia (one article), Portugal (one article), Canada (one article), Sri Lanka (one article), German (one article), Australia (two article), Amerika (one article), Vietnam (one article), England (one article), China (one article). The results of those 11 articles were summarized as follows in Table 1 (see in Appendix).

The results showed several mental health literacy implementations for adolescents. There were similarities and differences in the implementation of adolescents in advance and developing countries. In advanced countries, the efforts of mental health literacy were done through programmed and timed preferences. The mental health literacy programs were promoted face to face by utilizing information technology for having a wider scope. It was well-planned, structured, and evaluated. The government attempted to promote mental health literacy efforts by involving many stakeholders. In the school domain, the mental health literacy curriculum had been integrated arranged for the school society, such as teachers, students, and parents. The given programs were such as to reduce stigma, to promote mental health science, and to improve mental health literacy, wellbeing, and emotional endurance. In developing countries, the efforts were such as: cooperating with stakeholders and evaluating the mental health literacy program 
reliability; involving many parties in the mental health literacy program, such as the central government, the local government, school, community, family, and individual. The applied programs in the developing countries had introductory natures to improve skills in recognizing mental health problems.

Similar mental health literacy programs in the advanced countries and developing countries were found to require a national scale policy and also cross-sector cooperation. The skills to recognize mental health problems, the intervention, and the useful results in the population were found equivalent to the adolescent population in other countries. However, there were differences in terms of prevention, identification, and intervention. The advanced countries had applied mental health literacy programs assisted by technology for a wider scope.

There were various strategies such as national strategy is done by: i) providing policy, fund [21]; implementing a preventive method, intervention, and national scale curation; providing national/province/municipality/regency mental health service call centers [22] that cooperate with a related institution, such as rehabilitation center, health institution, and psychiatric hospital; ii) workshop with the concerning stakeholders regularly; iii) conducting transformative research for mental health problems, iv) creating synergy with experts or cross-sector for the sake of mental health literacy program (Anti stigma) [23], [24]. When the stakeholders are not involved in planning the service that could influence them, the service might be failed to meet their needs. The community-based strategy could be done by i) involving adolescents as the agents of change [25], ii) using technology such as the Internet to do campaign (by using the web, playing a game such as moving stories, peer support establishment) [26]; and iii) using the Internet to provide prevention and intervention [27], [28]. School-based strategy The importance of a strategy to improve mental health literacy by targeting students [29], [30]. It is since education is important to improve mental health science, such as depression [31], reduce stigma, and improve access to the care [32]. This strategy could be done by i) creating a synergy with a school-based mental health literacy program, ii) reporting mental health cases in the educational unit, iii) promoting mental health by using games [33], group dynamics, modeling, kinds of music, video, photo novel, and comic [34], iv) forming a University level agency to find out the report of mental health disorders at each educational unit to be followed up, and v) providing an integrated program about school-based anti-stigma in their mental health literacy programs [35]. Individual-based strategy. It could be done by i) providing individual training to recognize problems and seek accessible assistance for the adolescents, ii) providing an introductory program, and improving self-capacity, such as improving personal awareness [36], self-respect training [37], and empathy training [38].

Mental health literacy is a new study area, especially in developing countries [39]. Mental health literacy is mostly ignored by several developing countries especially in their suburban area. Those areas are sometimes having limited mental health service access [40]. Based on data, developing countries such as Indonesia, Vietnam and Sri Lanka use different methods in applying mental health literacy. In Indonesia, the implementation of mental health literacy using coordination with local stakeholders. In Vietnam, the bond of the parents with the adolescents within South East Asian Culture context became an effective medium to promote adolescents' mental health. Family support can be done by optimizing family functions to help adolescence. Empowment is a core concep of the World Health Organization's (WHO) vision of health promotion. Empowerment in mental health field is recognized as a key priority of the WHO Mental Health Declaration for Europe and the WHO European Mental Health Action Plan for persons mental health issues and their family caregivers [41]. With a high rate of mental health problems in the world, there would be spaces to improve mental health literacy in developing countries. Low mental health literacy would hinder effective medication for those who need it. It would contribute significantly toward the disease load in the society where mental health care was limited and not strengthened by economic equality [42].

Meanwhile, in advanced countries such as Australia, Canada, German, England and China, mental health literacy had been done in systematical manners. The advance countries used the educational setting and educational intervention in reducing the stigma, promoting health mental science, and mental health literacy. The programs such as short term program to improve mental health literacy at school, used internet based mental health programs, integrated science education outreach program delivered the school based anti stigma of mental health. School is a protective factor to improve and promote mental health. School counselors are the professionals within the school who are tehe best equipped to deal with the mental health concerns of students and particulary the protective factors affecting those concerns [43]. Future research to involve community groups such as school, communities and strenghten the mental health of individuals through mental health literacy programs.

\section{CONCLUSION}

Adolescents need to have mental health literacy. Mental health literacy is a new study area, especially in developing countries. The results of the literature reviews showed mental health literacy for 
adolescents across the countries. The efforts were national, community, and individual in nature. The importance of the synergy of these various elements to improve mental health literacy to form a resilient country.

\section{ACKNOWLEDGEMENT}

The researcher would like to thank the Education funding agency (LPDP) for providing financial assistance during the study. Thanks are also given to the Airlangga University Psychology Doctoral Program for all the support.

\section{REFERENCES}

[1] D. Fuhrmann, L. J. Knoll, and S. J. Blakemore, "Adolescence as a Sensitive Period of Brain Development," Trends Cogn. Sci., vol. 19, no. 10, pp. 558-566, 2015, doi: 10.1016/j.tics.2015.07.008.

[2] E. Bagalman and A. Napili, "Prevalence of mental Illness in the United States: Data sources and estimates," Fed. Programs Support. People with Serious Ment. Illn. An Assess., pp. 73-85, 2015, [Online]. Available: \%0Awww.crs.gov.

[3] E. Hoge, D. Bickham, and J. Cantor, "Digital media, anxiety, and depression in children," Pediatrics, vol. 140, no. November 2017, pp. S76-S80, 2017, doi: 10.1542/peds.2016-1758G.

[4] B. Keles, N. McCrae, and A. Grealish, "A systematic review: the influence of social media on depression, anxiety and psychological distress in adolescents," International Journal of Adolescence and Youth, vol. 25, no. 1. pp. 7993, 2020, doi: 10.1080/02673843.2019.1590851.

[5] F. J. Carod-Artal, "Social determinants of mental health," Glob. Ment. Heal. Prev. Promot., pp. 33-46, 2017, doi: 10.1007/978-3-319-59123-0_4.

[6] D. Eisenberg, E. Golberstein, and J. Hunt, "Mental Health and Academic Success in College," The B.E. Journal of Economic Analysis \& Policyno, vol. 9, no. 1, pp. 1-40, 2009, doi: 10.2202/1935-1682.2191.

[7] Mental Health Foundation, Fundamental Facts About Mental Health 2016. London, 2016.

[8] S. Asyanti and U. Karyani, "Mental Health Literacy Among Youth in Surakarta," vol. 133, pp. 12-16, 2018, doi: 10.2991/acpch-17.2018.24

[9] Q. C. Nguyen Thai and T. H. Nguyen, "Mental health literacy: Knowledge of depression among undergraduate students in Hanoi, Vietnam," Int. J. Ment. Health Syst., vol. 12, no. 1, pp. 1-8, 2018, doi: 10.1186/s13033-0180195-1.

[10] L. T. Lam, "Mental health literacy and mental health status in adolescents: A population-based survey," Child Adolesc. Psychiatry Ment. Health, vol. 8, no. 1, pp. 1-8, 2014, doi: 10.1186/1753-2000-8-26.

[11] K. Chisholm, P. Patterson, C. Torgerson, E. Turner, D. Jenkinson, and M. Birchwood, "Impact of contact on adolescents' mental health literacy and stigma: The schoolspace cluster randomised controlled trial," BMJ Open, vol. 6, no. 2, pp. 1-11, 2016, doi: 10.1136/bmjopen-2015-009435.

[12] U. R. Attygalle, H. Perera, and B. D. W. Jayamanne, "Mental Health Literacy in Adolescents: Ability to Recognise Problems, Helpful Interventions and Outcomes," Child Adolesc. Psychiatry Ment. Health, vol. 11, no. 1, pp. 1-8, 2017, doi: 10.1186/s13034-017-0176-1.

[13] A. Gulliver et al., "Internet-based interventions to promote mental health help-seeking in elite athletes: An exploratory randomized controlled trial," J. Med. Internet Res., vol. 14, no. 3, 2012, doi: 10.2196/jmir.1864.

[14] M. Marcus and H. Westra, "Mental health literacy in Canadian young adults: Results of a national survey," Can. J. Community Ment. Heal., vol. 31, no. 1, pp. 1-15, 2012, doi: 10.7870/cjcmh-2012-0002.

[15] X. Y. Zhuang, D. F. K. Wong, C. W. Cheng, and S. M. Pan, "Mental health literacy, stigma and perception of causation of mental illness among Chinese people in Taiwan," Int. J. Soc. Psychiatry, vol. 63, no. 6, pp. 498-507, 2017, doi: 10.1177/0020764017719303.

[16] G. Rafal, A. Gatto, and R. DeBate, "Mental health literacy, stigma, and help-seeking behaviors among male college students," J. Am. Coll. Heal., vol. 66, no. 4, pp. 284-291, 2018, doi: 10.1080/07448481.2018.1434780.

[17] S. Evans-Lacko, E. Brohan, R. Mojtabai, and G. Thornicroft, "Association between public views of mental illness and self-stigma among individuals with mental illness in 14 European countries," Psychol. Med., vol. 42, no. 8, pp. 1741-1752, 2012, doi: 10.1017/S0033291711002558.

[18] S. Kraus, S., Breier, and M. y Dasí-Rodríguez, "El arte de elaborar una revisión bibliográfica sistemática en la investigación sobre el espíritu empresarial," Int. Entrep. Manag. J., vol. 16, pp. 1023-1042, 2020.

[19] A. Petrosino, C. Morgan, T. A. Fronius, E. E. Tanner-Smith, and R. F. Boruch, "Interventions in Developing Nations for Improving Primary and Secondary School Enrollment of Children: A Systematic Review," Campbell Syst. Rev., vol. 8, no. 1, 2012, doi: 10.4073/csr.2012.19.

[20] L. A. Maggio, J. L. Sewell, and A. R. Artino, "The Literature Review: A Foundation for High-Quality Medical Education Research,” J. Grad. Med. Educ., vol. 8, no. 3, pp. 297-303, 2016, doi: 10.4300/JGME-D-16-00175.1.

[21] B. H. Liese, R. S. F. Gribble, and M. N. Wickremsinhe, "International funding for mental health: A review of the last decade," Int. Health, vol. 11, no. 5, pp. 361-369, 2019, doi: 10.1093/inthealth/ihz040.

[22] R. Ramchand, L. Jaycox, and P. Ebener, "Suicide Prevention Hotlines in California: Diversity in Services, Structure, and Organization and the Potential Challenges Ahead," Suicide Prev. Hotlines Calif. Divers. Serv. Struct. Organ. Potential Challenges Ahead, 2017, doi: 10.7249/rr1497.

[23] S. Knaak, Stephanie; Patten, J. D. Livingston, S. Patten, S. Knaak, S. Francisco, and M. Pietrus, "Building and 
Delivering Successful Anti-stigma Programs for Healthcare Providers. Results of a Qualitative Study," Ment. Heal. Comm. Canada, vol. 83, no. September, pp. 1-54, 2013, [Online]. Available: http://www.mentalhealthcommission.ca.

[24] S. E. Hanisch, C. D. Twomey, A. C. H. Szeto, U. W. Birner, D. Nowak, and C. Sabariego, "The effectiveness of interventions targeting the stigma of mental illness at the workplace: A systematic review," BMC Psychiatry, vol. 16, no. 1, pp. 1-11, 2016, doi: 10.1186/s12888-015-0706-4.

[25] P. Toolkit, Y. People, and I. A. Health, “Advocating for Change for Adolescents!," Wellbeing. Geneva World Heal. $\begin{array}{llll}\text { Organ., no. } & \text { May, } & \text { [Online]. } & \text { Available: }\end{array}$ http://www.who.int/pmnch/knowledge/publications/advocacy_toolkit.pdf.

[26] H Christensen and K Griffiths "The Internet and Mental Health Literacy," Australian \& New Zealand Journal of Psychiatry, vol. 34, no. 6, pp. 975-979, 2000, doi: 10.1080/000486700272.

[27] Á. Jiménez-Molina, P. Franco, V. Martínez, P. Martínez, G. Rojas, and R. Araya, "Internet-Based Interventions for the Prevention and Treatment of Mental Disorders in Latin America: A Scoping Review," Front. Psychiatry, vol. 10, no. 664, pp. 1-17, 2019, doi: 10.3389/fpsyt.2019.00664.

[28] S. Y. Lee, M. S. Kim, and H. K. Lee, "Prevention Strategies and Interventions for Internet Use Disorders Due to Addictive Behaviors Based on an Integrative Conceptual Model," Curr. Addict. Reports, vol. 6, no. 3, pp. 303-312, 2019, doi: 10.1007/s40429-019-00265-z.

[29] N. J. Reavley, T. V. McCann, and A. F. Jorm, "Mental Health Literacy in Higher Education Students," Early Interv. Psychiatry, vol. 6, no. 1, pp. 45-52, 2012, doi: 10.1111/j.1751-7893.2011.00314.x.

[30] R. Miles, L. Rabin, A. Krishnan, E. Grandoit, and K. Kloskowski, "Mental health literacy in a diverse sample of undergraduate students: demographic, psychological, and academic correlates," BMC Public Health, vol. 20, no. 1, pp. 1-13, 2020, doi: 10.1186/s12889-020-09696-0.

[31] C. Lauber, V. Ajdacic-Gross, N. Fritschi, N. Stulz, and W. Rössler, "Mental Health Literacy in an Educational Elite - An Online Survey Among University Students,” BMC Public Health, vol. 5, no. 44, pp. 1-9, 2005, doi: 10.1186/1471-2458-5-44.

[32] J. Knickman et al., "Improving Access to Effective Care for People Who Have Mental Health and Substance Use Disorders: A Vital Direction for Health and Health Care," NAM Perspect., vol. 6, no. 9, 2016, doi: $10.31478 / 201609 \mathrm{v}$

[33] A. Tuijnman, M. Kleinjan, E. Hoogendoorn, I. Granic, and R. C. M. E. Engels, "A Game-based school program for mental health literacy and stigma regarding depression (moving stories): protocol for a randomized controlled trial," J. Med. Internet Res., vol. 21, no. 3, pp. 1-12, 2019, doi: 10.2196/11255.

[34] A. C. Yulianti, Padmi Dhyah; Suyati, Tri;Rakhmawati, Ellya;Sagala, "The Design of Comic Development as Effort to Prevent Early,” vol. 287, no. Icesre 2018, pp. 244-248, 2019, doi: 10.2991/icesre-18.2019.53.

[35] M. Koller, S.-P. Chen, J. Ledoux-Moshonas, A. D 'alessio, J. Deslauriers, and H. Stuart, "Opening Minds in High School: Results of a Contact-Based Anti-Stigma Intervention 2 Opening Minds: Changing How We See Mental Illness," no. March, 2013, [Online]. Available: www.mentalhealthcommission.ca.

[36] F. Hatami, L. Ghahremani, M. Kaveh, and S. Keshavarzi, "The Effect of Self-Awareness Training with Painting on Self-Efficacy among Orphaned Adolescents,” Pract. Clin. Psychol., vol. 4, no. 2, pp. 89-96, 2016, doi: 10.15412/j.jpcp.06040203.

[37] T. Ramezani, G. Sharifirad, Z. Gharlipour, and S. Mohebi4, "Effect of Educational Program based on Self-Efficacy Theory on Improvement of Mental Health in Hemodialysis Patients," Heal. Educ. Heal. Promot., vol. 5, no. 2, pp. 67-79, 2017.

[38] A. Furnham and P. Sjokvist, "Empathy and Mental Health Literacy," HLRP Heal. Lit. Res. Pract., vol. 1, no. 2, pp. e31-e40, 2017, doi: 10.3928/24748307-20170328-01.

[39] H. M. Dang, B. Weiss, T. Lam, and H. Ho, "Mental health literacy and intervention program adaptation in the internationalization of school psychology for Vietnam," Psychol. Sch., vol. 55, no. 8, pp. 941-954, 2018, doi: 10.1002/pits.22156.

[40] M. Kermode, K. Bowen, S. Arole, K. Joag, and A. F. Jorm, "Community beliefs about treatments and outcomes of mental disorders : A mental health literacy survey in a rural area of Maharashtra, India," Public Health, vol. 123, no. 7, pp. 476-483, 2009, doi: 10.1016/j.puhe.2009.06.004.

[41] M. Kageyama, Y. Nakamura, S. Kobayashi, K. Yokoyama, "Osaka University Knowledge Archive: OUKA," Journal of Psychiatric and Mental Health Nursing, vol. 23, pp. 521-531, 2016, doi: 10.1111/jpm.12333.

[42] K. A. Ganasen, S. Parker, C. J. Hugo, D. J. Stein, R. A. Emsley, and S. Seedat, "Mental Health Literacy: Focus on Developing Countries," African J. Psychiatry (South Africa), vol. 11, no. 1, pp. 23-28, 2008, doi: 10.4314/ajpsy.v11i1.30251.

[43] J. V Carney, H. Kim, R. J. Hazler, and X. Guo, "Protective Factors for Mental Health Concerns in Urban Middle School Students : The Moderating Effect of School Connectedness," Professional School Counseling, vol. 21, no. 1, pp. 1-9, 2018, doi: 10.1177/2156759X18780952.

[44] H. Brooks et al., "Improving mental health literacy among young people aged 11-15 years in Java, Indonesia: Codevelopment and feasibility testing of a culturally-appropriate, user-centred resource (IMPeTUs) - A study protocol," BMC Health Serv. Res., vol. 19, no. 484, pp. 1-9, 2019, doi: 10.1186/s12913-019-4328-2.

[45] P. Dias, "Is It Possible to 'Find Space for Mental Health' in Young People? Effectiveness of a School-Based Mental Health Literacy Promotion Program," Int. J. Environ. Reseacrh Public Heal., vol. 15, no. 7, pp. 1-12, 2018, doi: 10.3390/ijerph15071426.

[46] L. L. Armstrong and K. Young, "Psychosocial Intervention students," Psychosoc. Interv., vol. 24, no. 2, pp. 83-87, 
2015, doi: 10.1016/j.psi.2015.05.002.

[47] G. Schomerus, M. C. Angermeyer, S. E. Baumeister, S. Stolzenburg, B. G. Link, and J. C. Phelan, "An Online Intervention Using Information on The Mental Health-Mental Illness Continuum to Reduce Stigma," Eur. Psychiatry, vol. 32, pp. 21-27, 2016, doi: 10.1016/j.eurpsy.2015.11.006.

[48] W. W. Mak, F. H. Chio, A. T. Chan, W. W. Lui, and E. K. Wu, "The Efficacy of Internet-Based Mindfulness Training and Cognitive-Behavioral Training with Telephone Support in The Enhancement of Mental Health among College Students and Young Working Adults: Randomized Controlled Trial," J. Med. Internet Res., vol. 19, no. 3, pp. 1-16, 2017, doi: 10.2196/jmir.6737.

[49] J. Yang, R. Lopez Cervera, S. J. Tye, S. C. Ekker, and C. Pierret, "Adolescent Mental Health Education InSciEd Out: A Case Study of an Alternative Middle School Population," J. Transl. Med., vol. 16, no. 1, pp. 1-10, 2018, doi: 10.1186/s12967-018-1459-x.

[50] H. T. L. Nguyen, K. Nakamura, K. Seino, and S. Al-Sobaihi, "Impact of parent-adolescent bonding on school bullying and mental health in Vietnamese cultural setting: Evidence from the global school-based health survey," BMC Psychol., vol. 7, no. 1, pp. 1-10, 2019, doi: 10.1186/s40359-019-0294-z.

[51] A. J. Morgan et al., "Does Mental Health First Aid Training Improve The Mental Health of Aid Recipients? The Training for Parents of Teenagers Randomised Controlled Trial," BMC Psychiatry, vol. 19, no. 1, pp. 1-14, 2019, doi: 10.1186/s12888-019-2085-8.

[52] C. Guo, G. Tomson, C. Keller, and F. Söderqvist, "Prevalence and correlates of positive mental health in Chinese adolescents," BMC Public Health, vol. 18, no. 1, pp. 1-11, 2018, doi: 10.1186/s12889-018-5133-2.

\section{APPENDIX}

Tabel 1. Final data

\begin{tabular}{|c|c|c|c|}
\hline AUTHORS & COUNTRY & SAMPLE & RESULTS \\
\hline Helen Brooks, & Indonesia & The respondents were & ts to support \\
\hline Irmansyah & & aged from $11-15$ & elf-management were the low- \\
\hline Irmansyah, Karina & & years old. $15-20$ & cost methods in which th \\
\hline Lovell, Ira Savitri, & & parents or child or & overcome the anxiety burden and depression \\
\hline Utomo, & & adolescent care with & among the children and adolescents. However, \\
\hline Prawira, & & depression & this is the unexplored location in Indonesia. \\
\hline Iskandar, & & anxiety. The workers & Cooperating with the local stakeholders, this \\
\hline Laoise Renwick, & & and teachers, in terms & research designed and evaluated the reliability \\
\hline Rebecca Pedley & & perspective & of the mental health literacy, anxiety, and \\
\hline Agustin Kusumayati & & about the intervention & depression that were focused on interactive \\
\hline & & design & \\
\hline
\end{tabular}

$\begin{array}{lll}\text { Campos L Dias P } & \text { Portugal } & 543 \text { students aged } 12 \\ \text { Duarte A, Veiga } & & 14 \text { years old. } \\ \text { E, Dias CC Palha F } & & \\ \text { [45] } & & 271 \text { learners } \\ \text { Lynne Armstrong } & \text { Canada } & \\ \text { and Kaitlyn Young } & & \\ \text { [46] } & \end{array}$

each professional group). The main informants' interviews consisted of $8-10$ people.

Udena Ruwindu Sri Lanka
Attygalle, Hemamali
Perera and Bernard
Deepal
Wanniarachchi
Jayamanne [12]
G. Schomerus, M.C. Germany
Angermeyer, S.E.
1002 adolescents aged 12 - 16 years old.

1679 people aged older than 15 years
"Finding out the space for mental health". It showed efficacy as a short-term promotion program to improve mental health literacy at schools.

Awareness, acknowledgment, and sharing appropriate science for adolescents were needed among the policymakers about the gaps of the learners' cognitions after the Senior High Schools.

Assessing mental health science in terms of what matters is preferred by the students to know after graduating from senior high school about mental health. Science transfer prevalence could assist the development of a theoretical framework to overcome the significant gaps in mental health necessity after graduating from senior high school by adjusting to their personalities.

The skills to recognize mental health problems, the intervention, and the useful results in the population were found equivalent to the adolescent population in other countries with several conditions. The main difference dealt with the identification and intervention in responding to the psychosis and social phobia sketch.

Attitudes toward individuals with a mental disorder could be improved by providing 


\begin{tabular}{lr}
\hline \multicolumn{2}{c}{ AUTHORS } \\
\hline Baumeister, \\
Stolzenburg, B.G \\
Link, J.C. Phelan \\
[47] \\
Winnie WS Mak \\
Floria HB Chio, \\
Amy TY Chan, \\
Wacy WS Lu \\
Ellery KY Wu [48]
\end{tabular}

Yang J, Lopez

Carvera R, Tye

SJ,Ekker SC,Pierret C. [49]

Hoang Thuy Linh

Nguyen, Keiko

Nakamura, Kaoruko

Seino, and Saber Al-

Sobaihi [50]

Katharine Chisholm,

Paul Patterson,

Carole Torgerson,

Erin Turner, David

Jenkinson, Max

Birchwood [11]

Amy J. Morgan,

Julie-Anne

A.

Fischer, Laura M.

Hart, Claire M.

Kelly , Betty A.

Kitchener,Nicola J.

Reavley, Marie B.

H. Yap, Stefan

Cvetkovski and

Anthony F. Jorm

[51]

Cheng Guo, Göran

Tomson, Christina

Keller and Fredrik

Söderqvis. [52]
America

350 students of $7-12$ grades.

380 learners

3331 learners of $8-12$

grades.

769 learners aged 12 13 years old.

384 parents and 384 adolescents aged 1215 were randomly taken to be treated by MHFA courses for 14 hours.

5399 students of $8-10$ grades.
RESULTS

information about mental disease continuum.

Both Internet-based mental health programs had potencies to improve mental health before the treatment. The improvement could be maintained in the third month of the follow-up. High crash frequency in this research showed the necessity of improvement for the futuristic technology based psychology program. The mental health professionals had to cooperate with information technology experts to improve web-based intervention personalization. It had a function to improve compliance.

The Integrated Science Education Outreach Program (InSciEd Out) delivered the schoolbased anti-stigma of mental health for learner groups with risk seven or eight.

The curriculum-based effort focused on mental health disease in an alternative, worth, and integrated school environment into the curriculum under InSciEd out-framework. The focus improvement of the society based program had the potentials to mediate the gaps in interpreting, to bring critical population toward clinical treatment in improving mental health.

The bond of the parents with the adolescents within South East Asian Culture context became an effective medium to promote adolescents' mental health.

The educational intervention seemed successful in reducing the stigma, promoting health mental science, improving mental health literacy, and improving the wellbeing and emotional endurance. A greater experiment is needed to confirm this result.

This research showed improvement in the mental health literacy of the trainees. However, there was no significant difference in adolescents' mental health and the given support for them by their parents when they had mental health problems.

China

Positive factors were found correlating to mental health in the regression model. They were such as sex types, economy, family, siblings, satisfaction toward personal appearance, physical activities, sleeping quality, stress, social trust, learning motivation, teacher's support, parents' supports, and school intimidation existence. 